\title{
Does mental health-related discrimination predict health service use 2 years later? Findings from an Australian national survey
}

Nicola J Reavley ${ }^{1}$, Principal Research Fellow

Amy J Morgan ${ }^{1}$, Senior Research Fellow

Dennis Petrie ${ }^{1,2}$, Principal Research Fellow

Anthony F Jorm ${ }^{1}$, Emeritus Professor

1 Centre for Mental Health, Melbourne School of Population and Global Health, The University of Melbourne, Australia

2 Centre for Health Economics, Monash Business School, Monash University, Australia

Corresponding author:

Nicola J Reavley, Centre for Mental Health, Melbourne School of Population and Global Health, 207

Bouverie Street, VIC 3010, The University of Melbourne, Australia.

Email: nreavley@unimelb.edu.au, P: +61 390357628 F: +61 393495815 


\title{
Does mental health-related discrimination predict health service use
}

\section{2 years later? Findings from an Australian national survey}

\author{
Abstract \\ Purpose \\ Stigma and discrimination are central concerns for people with mental health problems. The aim of \\ the study was to carry out a follow-up survey of a national survey of experiences of avoidance, \\ discrimination and positive treatment in people with mental health problems in order to explore \\ how those experiences relate to health service use.
}

\section{Methods}

In 2017, telephone interviews were carried out with 655 Australians aged 18+, who had participated in a 2014 survey and reported a mental health problem or scored highly on a symptom screening questionnaire. Questions covered mental health, disclosure, health service utilisation, and experiences of avoidance, discrimination and positive treatment in a variety of different settings. Regression analyses were used to assess the extent to which count of settings of experiences of avoidance, discrimination or positive treatment at baseline (2014) or follow-up (2017) predicted health service use at follow-up.

\section{Results}

An increase in past experiences of discrimination was associated with a greater number of visits to hospital or specialist doctors and an increase in positive treatment was associated with a greater number of visits to a mental health professional. Increases in both positive and negative experiences were associated with greater healthcare costs, but the costs were greatest for discrimination at follow-up (concurrent discrimination), primarily due to the cost of nights in hospital. 


\section{Conclusions}

While both discrimination and positive treatment are associated with greater healthcare costs, concurrent experiences were shown to be more important correlates of health service use than past experiences. Moreover, those in supportive environments may be more willing to engage in earlier evidence-based treatment for mental health problems. 


\section{Introduction}

People with mental health problems commonly face stigma and discrimination. In many countries, considerable research effort has gone into exploring negative attitudes and behaviours and their impacts on people with mental health problems $[1,2]$. In recent years, stigma-related research has seen a shift from studies of attitudes towards people with mental health problems towards those that attempt to explore the experiences of people with these problems. Typically, such studies assess experiences in those registered with health services and find high levels of both anticipated and experienced discrimination [1-4]. A small number of studies have also assessed experiences of discrimination in general populations, finding rates of unfair treatment ranging between $16 \%$ and $37 \%$, depending on time period, setting and life domain [5-7]. However, these studies have not incorporated detailed exploration of the nature of these experiences and their impacts and, importantly, no population-based studies have systematically assessed experiences of positive treatment, although Thornicroft et al. have assessed these in selected service users with schizophrenia [2].

Evidence suggests that adverse impacts of discrimination include social isolation, reduced ability to achieve vocational and educational goals, limited access to housing, delayed professional helpseeking and access to appropriate treatment, which, due to more severe symptoms, may result in the use of relatively expensive acute services rather than primary care services [8-10]. Therefore, discrimination may carry economic costs to individuals and to society as a whole.

Two studies have attempted to explore the impact of discrimination on healthcare costs. EvansLacko et al. [11] used data from two cross-sectional UK studies (including the Viewpoint survey) to examine experiences of discrimination due to mental illness in the domains of personal relationships, community activities, and health care, and how this discrimination related to patterns of service use. Their findings showed that the cost of health services for individuals who reported previous experiences of discrimination in a healthcare setting was almost twice as high as for those 
who did not report any discrimination during the last 12 months and this difference was maintained after controlling for symptoms and functioning. Individuals who reported any discrimination in a healthcare setting had, on average, UK pound 434 higher costs associated with health service use. Also using data from the Viewpoint survey, Osumili et al. [12] found that individuals who reported experiences of discrimination in healthcare settings had higher costs of health service compared with those who did not. They also found that people who reported experiences of discrimination in relationships had higher healthcare costs than those who did not.

In summary, there is limited research suggesting that mental health discrimination experienced in healthcare or personal relationships is associated with higher healthcare costs. However, both these studies were cross-sectional, and may have included biased samples due to self-selection and low response rates. Moreover, only participants registered with secondary mental health services were involved, thereby precluding the assessment of the links between discrimination and healthcare costs for those disengaged with services. Additionally, there was no assessment of the links between positive experiences and healthcare costs. Therefore, there is a need for prospective studies that include more detailed exploration of these issues.

In 2014, we conducted a survey involving computer-assisted telephone interviews (CATI) with a national sample of 5220 members of the Australian community aged 18+, 1381 of whom reported a mental health problem or scored highly on a symptom screening questionnaire [13]. These respondents answered questions covering experiences of avoidance, discrimination and positive treatment in family, workplace, healthcare and community settings. At the end of the interview, 92\% of respondents who answered these questions agreed to be re-contacted, offering the opportunity for a follow-up study to prospectively explore the impacts of experiences of avoidance, discrimination and positive treatment in people with mental health problems. The aim of the study was to examine the association of past and present avoidance, discrimination or positive treatment on healthcare utilisation and costs. 


\section{Methods}

The follow-up survey involved computer-assisted telephone interviews (CATI) with respondents involved in a previous survey who had either scored in the 'high' range on the K6 (equal to or above 19) or who reported having one of the following mental health problems: depression/major depression, attempted suicide or self-harm , anxiety/anxiety disorder, post-traumatic stress disorder/PTSD, agoraphobia, panic disorder, obsessive-compulsive disorder/OCD, social phobia, generalised anxiety disorder/GAD, eating disorder/ anorexia/bulimia, schizophrenia/paranoid schizophrenia, schizoaffective disorder , psychosis/psychotic, bipolar/bipolar disorder/manicdepressive disorder, mental illness, personality disorder/borderline personality disorder, attention deficit-hyperactivity disorder/ADHD, Autism/Asperger's and nervous breakdown. See Reavley and Jorm [13] for further details. These respondents had answered a series of questions about how people had reacted to them as a result of any emotional or mental health problems they had experienced in the previous 12 months, notably about their experiences of avoidance and discrimination (which was and positive treatment (with discrimination-defined as people-being treated unfairly because they are seen as different from others). They were also asked if they had been treated more positively because of their emotional or mental health problems. At the end of that interview, respondents were asked whether they we willing to be recontacted for a follow-up survey on the same topic, with 1286 (92\%) indicating that they were.

All respondents who provided a mobile phone number in the 2014 survey were sent a pre-approach text message and all those who provided an email address were sent a pre-approach email. Call procedures for the survey featured: (1) Call attempts placed over different days of the week and times of day; (2) An unlimited number of calls to the person until the survey was closed; (3) Limited call initiation during business hours except by appointment; and (4) Late shifts dialling to landline numbers identified as Western Australia (which has a different time zone). Interviews were 
conducted between March and April 2017. Ethics approval was obtained from the University of Melbourne Human Research Ethics Committee.

\section{Survey interview}

Respondents were taken through the 12-month version of the Kessler 6 (K6) mental health symptom screening questionnaire [14]. This questionnaire asks participants to think about one month in the last 12 months when they were most depressed, anxious, or emotionally stressed. Respondents were also asked whether, over the last 12 months, they had experienced any sort of mental health problem (defined in the preamble to the question in the following way: "a period of weeks or more when you are feeling depressed, anxious, or emotionally stressed, and these problems are interfering with your life. Mental health problems could include, for example, depression, anxiety disorders, eating disorders, schizophrenia, bipolar disorder, or personality disorders"). Those respondents who answered yes to this question were then asked what they thought the problem was, if they had been given a diagnosis (and what this was) and if they had received treatment (and what this was). At this point, survey respondents were divided into two groups: (1) those who scored in the 'high' range on the K6 (equal to or above 19) or who reported having had one of the mental health problems listed above; and (2) those who did not meet these criteria.

Those in the first group were then asked the following questions about their experiences of avoidance, discrimination and positive treatment (with a past 12-month timeframe specified in the introduction to this section of the questionnaire): "Have any of your friends avoided you because of the emotional or mental health problems you have told me about?"; "Have any of your friends discriminated against you in other ways because of these problems?"; "Can you please describe what happened?"; "Have any of your friends treated you more positively because of these problems?"; and "Can you please describe what happened?". The same questions were asked for the following people or situations: spouse or intimate partner, other members of the family, people in the workplace, looking for work, people in the place of education, health professionals (with an 
additional question on type of professional), other people in the community or neighbourhood, other people and other situations. For people or situations in the categories of 'looking for work', 'health professionals', 'other people' and 'other situations', the question relating to avoidance was omitted. These questions were specifically designed for this survey as existing scales were either too long for the use in a CATI or did not cover positive treatment in every life domain [15].

\section{Healthcare utilisation}

Questions about respondents' health service use were based on those included in the Household, Income and Labour Dynamics in Australia (HILDA) survey (Wave 13), a large nationallyrepresentative panel of Australian households focussing on a wide range of social, demographic, health, and economic topics (https://melbourneinstitute.unimelb.edu.au/hilda) that commenced in 2001 [16]. These questions explored whether respondents had seen their GP or family doctor and, if so, the number of visits in the previous 12 months and whether there were out-of-pocket expenses; whether they had seen other healthcare providers and, if so, the number of visits to any of the following: hospital doctor, specialist doctor, psychiatrist, psychologist, counsellor, community mental health team; whether respondents had been in hospital for overnight stay and, if so, the number of admissions and the number of nights in hospital. Respondents were also asked to indicate whether they had a healthcare card (indicating a low income).

\section{Disclosure}

Respondents were asked the following: "Have you told the following people about these emotional or mental health problems that you have had in the last 12 months?" The questions covered the following people: Friends, spouse or intimate partner, other family members, your supervisor or boss at work, other people in your workplace, teachers or lecturers in your place of education, other people in your place of education, health professionals who are not involved in treating these problems and people in your neighbourhood or community'. Other than for intimate partner or supervisor (for which possible responses were 'Yes' or 'No'), possible response categories were: 'Yes, 
told everybody', 'Yes, told some people' or 'No, not told anybody'. For each setting, a dichotomous variable (told everybody/told some people vs told nobody) was created and a count of the number of settings in which a person had disclosed was calculated.

Further questions were asked about the impact of avoidance, discrimination or positive treatment on respondent mental health and on workplace participation. Data relating to this section of the survey will be reported elsewhere. The average interview length was 18 minutes.

\section{Statistical analysis}

Descriptive data were analysed using percent frequencies.

Logistic regression analyses were used to determine the relationships between past and current avoidance, discrimination or positive treatment and whether respondents had seen their family doctor, other healthcare providers or had been admitted to hospital. The following count of settings variables were simultaneously entered into the model: avoidance at baseline, avoidance at followup, discrimination at baseline, discrimination at follow-up, positive treatment at baseline, positive treatment at follow-up. The following covariates were also entered: age, gender, level of education, work status, marital status, language spoken at home, baseline K6 score, mental disorder (depression, anxiety, bipolar, other), whether the respondents had received treatment at baseline, count of disclosure and whether the respondents had a healthcare card (other than for admission to hospital).

Similarly, negative binomial regression analyses were used to explore the marginal effects of experiences of avoidance, discrimination or positive treatment at baseline and follow-up on the number of visits to the following: family doctor, hospital and specialist doctors (combined), mental health professionals and number of nights in hospital at follow-up. All regressions controlled for the same covariates as those listed above. Due to the number of statistical tests carried out, we used the more conservative $p<0.01$ as the significance level. 
The estimated marginal effects from the negative binomial regression analyses where then combined with unit costs for each healthcare use (in Year 2017 Australian dollars) to estimate the change in the average annual healthcare costs to the government associated with a one unit increase in count of avoidance, discrimination or positive treatment. Units costs were as follows: family doctor $\$ 37.05$; specialist doctor $\$ 43.00$; mental health professional $\$ 52.95$; and cost per night in hospital \$2062. These were based on the Australian Medicare Benefits Schedule (MBS) fees [17] which are the fees payed to providers by the Australian government for these services (out-ofpocket costs are excluded) and the Australian National Hospital Cost Data Collection [18] which reports the average cost per night in hospital and where needed converted into 2017 Australian dollars using the health consumer price index [19]. All analyses were performed using Intercooled Stata 14 (StataCorp LP, Texas, USA).

\section{Results}

Overall, 655 interviews were completed (from a usable sample of 1076 respondents), resulting in a sample yield of $61 \%$ and an American Association of Public Opinion Research (AAPOR) response rate of 55.1\%. Statistically significant predictors of not completing the follow-up interview included younger age (18-29), never being married, speaking a language other than English at home and having a high $\mathrm{K} 6$ score.

Among those who participated in the follow-up survey, 417 (64\%) had an in-scope mental health problem or a K6 score of 19 or more at follow-up, 325 (50\%) had received a diagnosis and 296 (45\%) had received treatment. See Table 1 for sample characteristics at follow-up. The mean (SD) counts at follow-up of avoidance, discrimination and positive treatment were 0.69 (0.99), $0.83(1.33)$ and 2.43 (1.85) respectively. Mean (SD) counts of healthcare use at follow-up were as follows: family doctor visits: 7.10 (9.52), hospital and specialist doctors (combined): 3.21 (9.04), mental health professionals: 4.15 (14.55) and number of nights in hospital: 1.79 (7.37). 


\section{Healthcare utilisation}

Table 2 gives the results of the logistic and negative binomial regression analyses of the associations between counts of avoidance, discrimination and positive treatment and healthcare utilisation. Concurrent (at follow-up) positive treatment was associated with higher odds of seeing healthcare providers other than family doctors.

Every one-unit increase in past (at baseline) discrimination was associated with just over one more visit to a hospital or specialist doctor. Every one-unit increase in current discrimination was associated with almost one more visit to a family doctor. Every one-unit increase in current positive treatment was associated with just over twice as many visits to a mental health professional.

Figure 1 gives the estimated change in annual health costs (in 2017) associated with a one unit increase in count of settings of avoidance, discrimination or positive treatment. First one can see that costs implications are mostly dominated by the nights in hospital. Increases in both supportive and negative experiences were associated with greater healthcare costs overall, but the costs were greatest for current discrimination, primarily due to the cost of nights in hospital. In relation to specific healthcare providers, past avoidance was associated with lower costs related to visits to a family doctor and a specialist or hospital doctor, whereas concurrent avoidance was associated with lower costs related to visits to a mental health professional. Past discrimination was only associatedion with minor additional costs while concurrent discrimination was associated with substantial increased costs mainly related to hospital stays. Past positive treatment was associated with lower current costs related to visits to a mental health professional but higher costs of nights in hospital, while current positive treatment was associated with higher costs related mainly to mental health professionals but had a more modest impact on hospital costs.

\section{Discussion}

This paper reports results of the first population-based follow-up survey to assess the impact of experiences of avoidance, discrimination and positive treatment in a broad range of settings on 
healthcare utilisation and costs. Concurrent experiences were shown to be more important correlates of health service use than past experiences, but past experience of discrimination was associated with a greater number of visits to hospital or specialist doctors. While the nature of positive treatment depends of the life domain, analysis of baseline data showed that this commonly involves the increased provision of emotional support (including being a good listener) and more social contact $[20,21]$. Discriminatory behaviour often involved a lack of understanding of the illness and its impact, reduced social contact and being denied opportunities (e.g. in the workplace).

Concurrent discrimination was associated with increased costs associated with visits to all professionals (particularly family doctors) and with the greatest healthcare costs, mostly due to nights in hospital. Past discrimination had some impact on costs related to contact with specialist or hospital doctors. Concurrent avoidance was associated with lower costs relating to visits to mental health professionals and concurrent positive treatment was associated with the greatest costs relating to visits to mental health professionals. Thus, while both discrimination and positive treatment seem to be associated with greater costs, those in supportive environments may be more likely to receive treatment from mental health professionals (thereby possibly avoiding or delaying the need to be hospitalised) while those in discriminatory environments may incur greater costs due to hospitalisation. In Australia, treatment by mental health professionals is funded under the Commonwealth's Better Access scheme, which allows psychologists and credentialed social workers and occupational therapists to claim Medicare rebates for patients referred by general practitioners [22]. Avoidance also appears to deter people from engaging in such treatment.

Because these analyses rely on data on concurrent experiences of positive treatment, discrimination and avoidance and healthcare usage this makes it difficult to draw conclusions about causality. It is possible that those with a greater health service use have more severe illness and are therefore more likely to experience discrimination and more likely to have positive experiences [23]. It is also possible that those who engage in psychological therapies show symptom improvements and are 
therefore less likely to experience discrimination. These findings provide support for antidiscrimination campaigns to have a greater focus on supportive behaviours rather than on what not to do.

To some extent these results align with those reported by Evans-Lacko et al. [11] who used UK survey data to examine the relationship between experiences of discrimination in healthcare settings and service use and showed higher costs for those with negative experiences, even after controlling for symptoms and functioning.

Considering the impact of past positive treatment, avoidance and discrimination on healthcare costs at follow-up, positive treatment was associated with greater healthcare costs (greater costs relating to hospital visit and lower costs relating to visits of mental health professionals). Discrimination at baseline was associated with lower costs overall but greater costs associated with each type of professional, while avoidance at baseline was associated with the lowest costs overall and lower costs of visits to specialists or hospital doctors and family doctors. These results suggest that negative experiences are associated with greater numbers of visits to mental health professionals while positive treatment is associated with fewer visits, presenting a somewhat different picture to the findings about concurrent experiences and healthcare usage described above. These findings possibly indicate that whether someone is treated negatively or positively treatment does not have significant long-term impact on healthcare costs, although it may mean that those who are treated positively are less likely to end up in acute care, which obviously has benefits in other areas of life.

\section{Strengths and limitations}

The study has several strengths. It is the first study to assess, at a population level, the long-term impact on health service use of mental health problem-related experiences of avoidance, discrimination and positive treatment in a wide range of domains. Interviews were used to gather reports of actual experiences of people rather responses to hypothetical situations. A further strength is the use of CATI random digit dialling to recruit the baseline sample, thereby reducing the 
likelihood of bias. However, the response rate was relatively low and may limit the generalisability of results. Moreover, those with high levels of psychological distress or who were unmarried were less likely to participate at follow-up. This may have led to an under-estimate of the experiences of avoidance and discrimination, as analysis of the baseline survey showed symptoms severity and being unmarried to be predictors of negative experiences but not positive treatment [23]. Future studies that involve linking to administrative health data would be useful to better understand the experience of non-responders.

A further limitation is that the small sample size means that we lack the statistical power to draw robust conclusions about the associated cost-simplications. There remains large uncertainty around the results with some clinically meaningful differences in healthcare use not reaching statistical significance. Future work should seek to address this. Finally, while there was broad coverage in the settings of discrimination and positive treatment, the costs were only considered at a health service level. Future work should explore costs and impacts on societal welfare in other areas, such as quality of life (including impacts on leisure activities), informal caregivers and the criminal justice system.

\section{Conclusions}

People with mental health problems in supportive rather than discriminatory environments may be more willing to engage in earlier evidence-based treatment for mental health problems, although the extent to which these experiences may delay rather than fully prevent the long-term impacts on healthcare costs is unclear.

\section{Funding}

The study was funded by the National Health and Medical Research Council.

\section{Conflict of interest}

On behalf of all authors, the corresponding author states that there is no conflict of interest. 


\section{References}

1. Lasalvia A, Zoppei S, Van Bortel T, Bonetto C, Cristofalo D, Wahlbeck K, Bacle SV, Van Audenhove C, van Weeghel J, Reneses B, Germanavicius A, Economou M, Lanfredi M, Ando S, Sartorius N, LopezIbor JJ, Thornicroft G (2013) Global pattern of experienced and anticipated discrimination reported by people with major depressive disorder: a cross-sectional survey. Lancet 381 (9860):55-62. doi:10.1016/S0140-6736(12)61379-8

2. Thornicroft G, Brohan E, Rose D, Sartorius N, Leese M (2009) Global pattern of experienced and anticipated discrimination against people with schizophrenia: a cross-sectional survey. Lancet 373 (9661):408-415. doi:10.1016/S0140-6736(08)61817-6

3. Angermeyer MC, Beck M, Dietrich S, Holzinger A (2004) The stigma of mental illness: patients' anticipations and experiences. Int J Soc Psychiatry 50 (2):153-162

4. Wahl OF (1999) Mental health consumers' experience of stigma. Schizophr Bull 25 (3):467-478 5. Alonso J, Buron A, Bruffaerts R, He Y, Posada-Villa J, Lepine JP, Angermeyer MC, Levinson D, de Girolamo G, Tachimori H, Mneimneh ZN, Medina-Mora ME, Ormel J, Scott KM, Gureje O, Haro JM, Gluzman S, Lee S, Vilagut G, Kessler RC, Von Korff M, World Mental Health C (2008) Association of perceived stigma and mood and anxiety disorders: results from the World Mental Health Surveys. Acta Psychiatr Scand 118 (4):305-314. doi:10.1111/j.1600-0447.2008.01241.x

6. Baldwin ML, Marcus SC (2006) Perceived and measured stigma among workers with serious mental illness. Psychiatr Serv 57 (3):388-392. doi:10.1176/appi.ps.57.3.388

7. Stuart H, Patten SB, Koller M, Modgill G, Liinamaa T (2014) Stigma in Canada: results from a rapid response survey. Can J Psychiatry 59 (10 Suppl 1):S27-33

8. Corker E, Hamilton S, Robinson E, Cotney J, Pinfold V, Rose D, Thornicroft G, Henderson C (2016) Viewpoint survey of mental health service users' experiences of discrimination in England 20082014. Acta Psychiatr Scand 134 Suppl 446:6-13. doi:10.1111/acps.12610 9. Corrigan P (2004) How stigma interferes with mental health care. Am Psychol 59 (7):614-625 
10. Link BG, Struening EL, Rahav M, Phelan JC, Nuttbrock L (1997) On stigma and its consequences: evidence from a longitudinal study of men with dual diagnoses of mental illness and substance abuse. J Health Soc Behav 38 (2):177-190

11. Evans-Lacko S, Clement S, Corker E, Brohan E, Dockery L, Farrelly S, Hamilton S, Pinfold V, Rose D, Henderson C, Thornicroft G, McCrone P (2015) How much does mental health discrimination cost: valuing experienced discrimination in relation to healthcare care costs and community participation. Epidemiology and psychiatric sciences 24 (5):423-434. doi:10.1017/\$2045796014000377 12. Osumili B, Henderson C, Corker E, Hamilton S, Pinfold V, Thornicroft G, McCrone P (2016) The economic costs of mental health-related discrimination. Acta Psychiatr Scand 134 Suppl 446:34-44. doi:10.1111/acps.12608

13. Reavley NJ, Jorm AF (2015) Experiences of discrimination and positive treatment in people with mental health problems: Findings from an Australian national survey. Aust N Z J Psychiatry 49 (10):906-913

14. Kessler RC, Green JG, Gruber MJ, Sampson NA, Bromet E, Cuitan M, Furukawa TA, Gureje O, Hinkov H, Hu CY, Lara C, Lee S, Mneimneh Z, Myer L, Oakley-Browne M, Posada-Villa J, Sagar R, Viana MC, Zaslavsky AM (2010) Screening for serious mental illness in the general population with the K6 screening scale: results from the WHO World Mental Health (WMH) survey initiative. Int J Methods Psychiatr Res 19 Suppl 1:4-22. doi:10.1002/mpr.310

15. Brohan E, Clement S, Rose D, Sartorius N, Slade M, Thornicroft G (2013) Development and psychometric evaluation of the Discrimination and Stigma Scale (DISC). Psychiatry Res 208 (1):33-40. doi:10.1016/j.psychres.2013.03.007

16. Summerfield M, Bevitt R, Freidin S, Hahn M, La N, Macalalad N, O'Shea M, Watson N, Wilkins R, M. W (2017) HILDA User Manual-Release 15. Melbourne Institute of Applied Economic and Social Research, Melbourne 
17. Australian Government Department of Health (2017) Quarterly Medicare Statistics. Department of Health. http://www.health.gov.au/internet/main/publishing.nsf/Content/Quarterly-MedicareStatistics. Accessed 1 November 20182019

18. Independent Hospital Pricing Authority (2017) National Hospital Cost Data Collection, public hospitals cost report, round 19 (financial year 2014-15). Independent Hospital Pricing Authority, Sydney

19. Australian Bureau of Statistics (2018) Australian Consumer Price Index: Concepts, Sources and Methods., vol Catalogue No.: 6401.0 Australian Bureau of Statistics, Canberra

20. Morgan AJ, Reavley NJ, Jorm AF, Beatson R (2016) Experiences of discrimination and positive treatment from health professionals: A national survey of adults with mental health problems. Aust N Z J Psychiatry 50 (8):754-762. doi:10.1177/0004867416655605

21. Morgan AJ, Reavley NJ, Jorm AF, Beatson R (2017) Discrimination and support from friends and family members experienced by people with mental health problems: findings from an Australian national survey. Soc Psychiatry Psychiatr Epidemiol 52 (11):1395-1403. doi:10.1007/s00127-0171391-z

22. Whiteford HA, Buckingham WJ, Harris MG, Burgess PM, Pirkis JE, Barendregt JJ, Hall WD (2014) Estimating treatment rates for mental disorders in Australia. Aust Health Rev 38 (1):80-85. doi:10.1071/AH13142

23. Reavley NJ, Morgan AJ, Jorm AF (2017) Predictors of experiences of discrimination and positive treatment in people with mental health problems: findings from an Australian national survey. Soc Psychiatry Psychiatr Epidemiol 52 (3):269-277. doi:10.1007/s00127-016-1301-9 


\section{Tables}

Table 1 Characteristics of the sample at follow-up ( $n=655)$

n

$\%$

Age

$18-29$

104

16

$30-59$

371

57

$60+$

180

27

Gender

Male

230

35

Female

423

65

Marital status

Married, de facto

350

53

Never married

161

25

Separated, divorced, widowed

144

22

Worked or volunteered

476

73

Below bachelor level

424

65

Not Aboriginal or Torres Strait Islander

633

97

Not born in Australia

122

19

English not first language

57

9

\section{Clinical characteristics}

K6 bands

6-10

119

18

11-15

198

30

$16-20$

183

28 


\section{Positive or negative treatment at baseline}

Experienced avoidance in any setting

\section{Positive or negative treatment at follow-up}

Experienced avoidance in any setting 
Table 2 Associations between baseline avoidance, discrimination or positive treatment and healthcare utilisation at follow-up

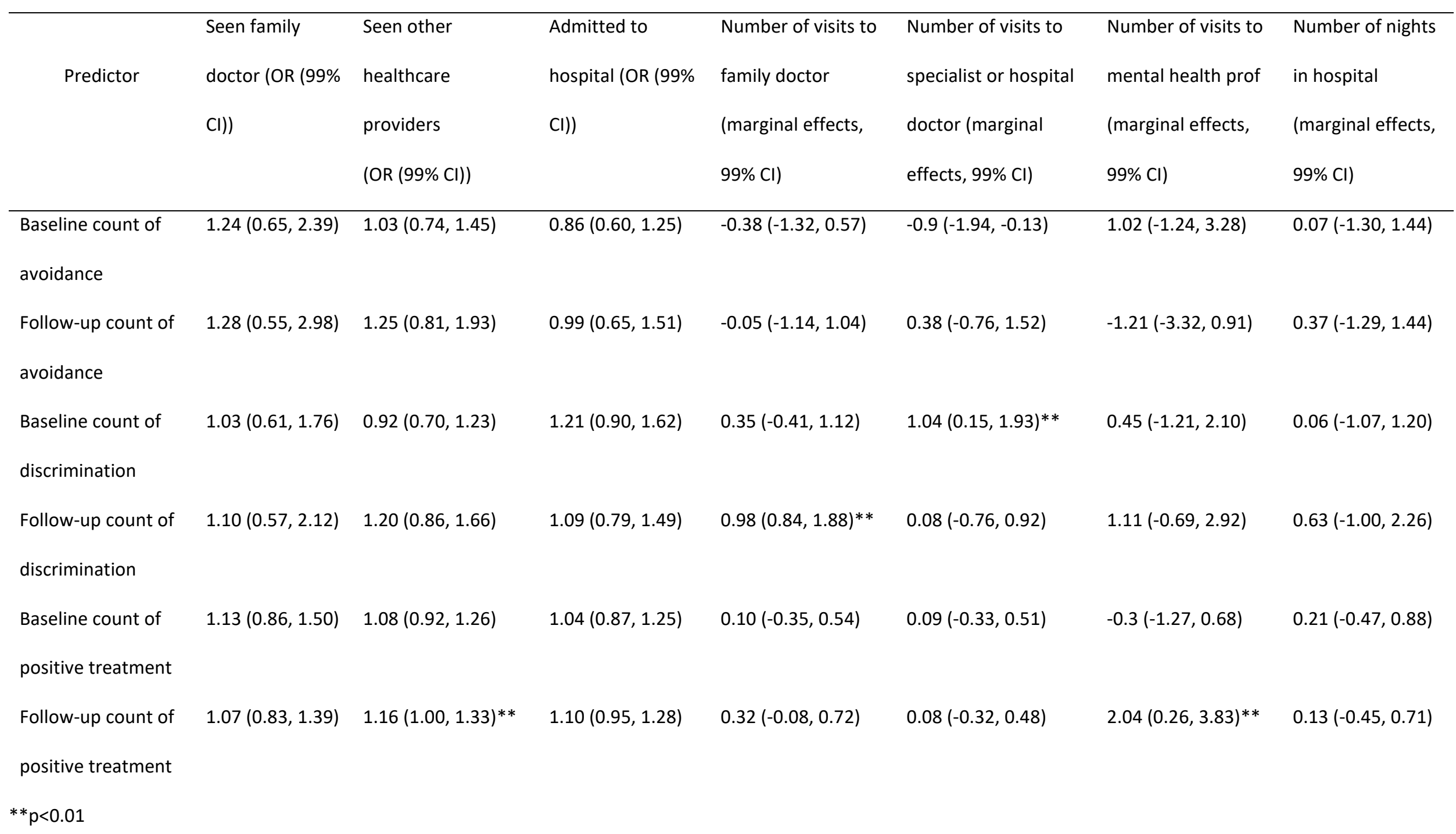




\section{Figures}

Figure 1 Annual Costs (\$2017) associated with a one unit increase in count of experiences at baseline and follow-up

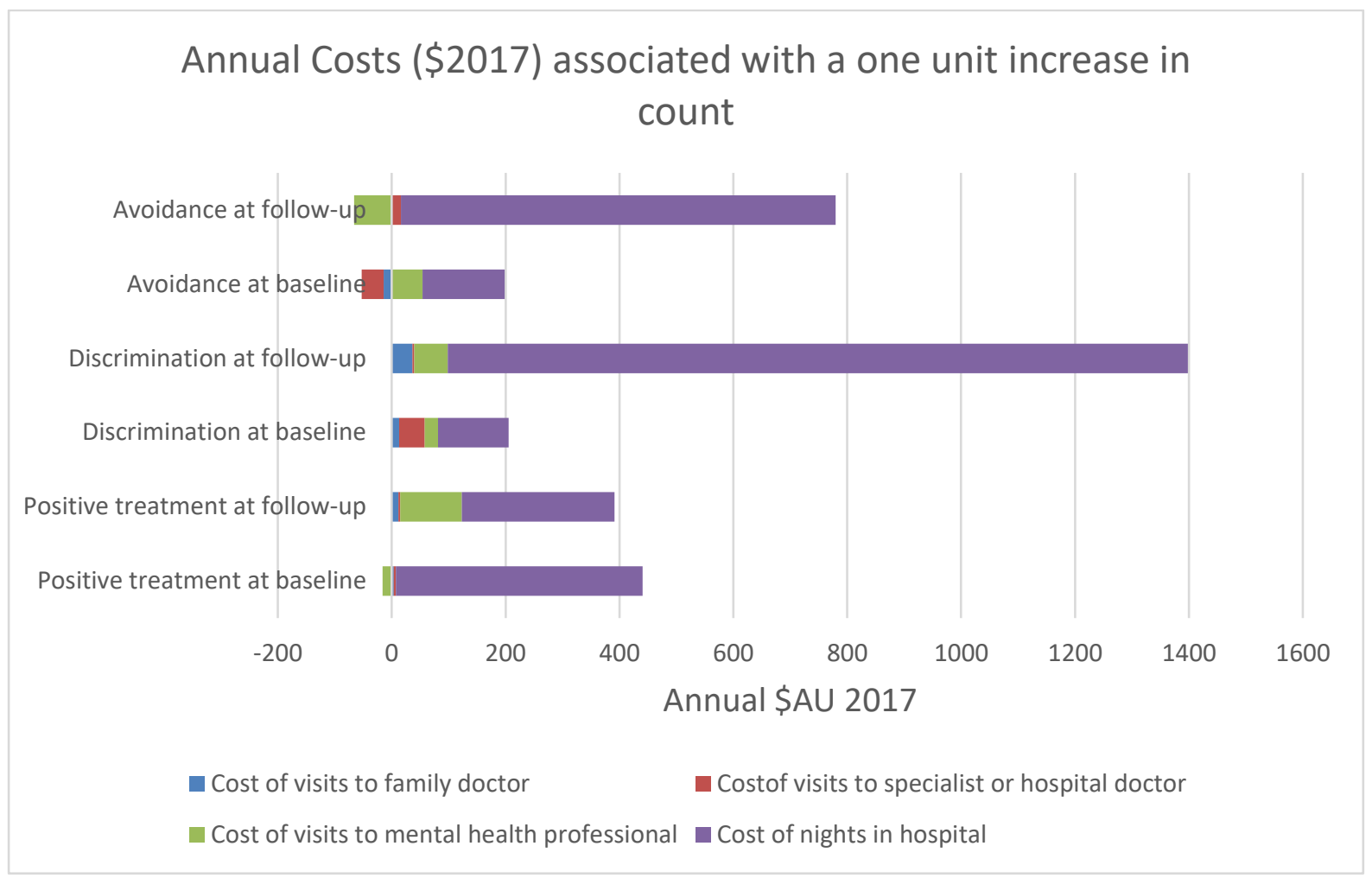

